$T D=4111$

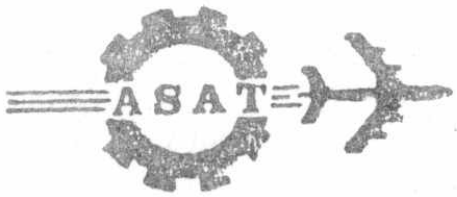

MILITARY TECHNICAL COLLEGE

CAIRO - EGYPT

\title{
EXPERIMENTAL INVESTIGATION ON A HEAT PIPE APPLIED TO AN AUTONOMOUS SOLAR REFRIGERATOR
}

A. R. El -Ghal ban

Lecturer, Mechanical Power Eng. Dept.

Faculty of Eng. \& l'echnology

Menoufia University

Shebin Elkom, EGYP'l

\section{ABSTRACT}

Heat rejection fxom an absorber by the vapor absorption refrigirator, is one of the most affecting processes on the system performance, specially for an autonomous operating system. A proposed absorber heat extracting technique is experimentally investigated to define the effect of the operating parameters upon the system performance. The proposed technique makes use of the gravity assisted heat pipe of a special form. It was observed that the evaporator filling ratio, for the heat pipe has an optimum value which depends mainly upon the difference between the operating temperatures for the evaporator and condenser.

\section{INTRODUCTION}

Solar cooling has its attraction specially for isolated regions, where the need for cooling comes in the same time with the solar energy exposure. Many investigations have been done in the subject of solar powered absorption refrigeration system as a field for solar energy utilization [1]. 'lhis system is the most convenient one for the developing countries due to its the simplicity in manufacturing, the independence of energy converting systems, and less need to inaintenance.

There are many difficulties that need to be overcome to achieve the proper operation of an autonomous continuous operating solar-powered absorption refrigerator. Heat rejection

from the system components, which must be cooled, could be taken as the most serious one. 'This problem arises from the difficulty to connect the system to any other energy or water recourses except solar radiation. This means that, the only available mechanism of heat transfer from the system components is the natural convection to air. This problem is more complicated by the absorber than that by the other system components such as dephlegmator and condenser. 'Ihe complexity arises from both the interdependence of heat and mass transport mechanisms by the absorption process. 
1

'I'hus, this paper deals with the cooling of the absorber by suggesting a new method to transport heat from it to the ambient air through heat pipes of special Eorm to fulfill the wequirements of both heat and mass liansfer processes. The proposed heat pipe is experimentally investigated regarding the above mentioned transport mechanisms. 'lhe experiments were carried out to determine the effect of the considered parameters upon the absorption process. These considered parameters are, the condenser temperature, evaporator temperature and the evaporator filling ratio (f). Moreover, the effect of the Elow rate of the liquid to be cooled on the heat pipe capability has been investigated. 'The evaporator filling ratio is the ratio of the refrigerant: volume within the evapoxator lo that volume required for the complete filling of the evaporator.

In addition, the obtained resulls are used to determine the optimum operating conditions of the absorption refrigeration cycle for the prescribed boundary conditions.'lhat leads to the determination of the required number of heat pipes for the absorber of a specified absorption cycle.

\section{THE PROPOSED SYSTEM}

\subsection{Requirements of the system}

This study was undertaken to develop a cooling system for the absorber of an autonomous solar powered absorption refrigerator and to investigate experimentally its behavior. Moreover, the study is extended to determine the capability of the proposed system. This technique should be able to transmit the heat generated by either falling film or arising bubble absorption concepts. For the falling film absorption the weak solution should be distributed uniformly upon the heat transport. surface within the absorber in the form of a liquid film with certain thickness. That creates enough surface area of contact between the absorbing solution and the absorbed vapor to insure proper absorption process. For the second absorption concept the cooling surface should be distributed uniformly within the absorber which would be filled with the absorbing solution. This insures uniform heat rejection from the entire absorber core, which leads to efficient absorber characteristics. In addition, the heat transfer process should be carried out with the minimum temperature difference between the solution which must be cooled and the heat sink which is the ambient air in the present application lo attain higher refrigerant concentration in the strong solution.

\section{2 Description of the proposed system}

In order to meet the above mentioned requirements for the absorber of an autonomous solar driven refrigerator, a proposed scheme for the cooling system to transmit heat naturally from the absorber core to the atmospheric air, is constructed, manufactured and tested in the present work. 
The function of this system depends upon heat pipe as a heat conducting medium. Heat pipes are used as an efficient heat transport mediun in many applications such as solar collectors, space heating, desalination, refrigeration power generation, cooling of electronic components and in the waste heat utilization, $\{2,3]$. Heat pipes are selected in this application because it has many advantages such as, its ability to transmit heat with zero temperature gradient inside it, simplicity in construction, robustability, reliability and independence of any moving parts.

The heat pipe consists essentially, as il is shown in Eigure(1), of an evacuated container within which an amount of working fluid is supplied. When the heat is applied on the evaporator, part of the liquid vaporizes, saturating the interior of the container with the pure vapor. As the wall of the condenser is cooled due to heat extraction, some of the vapor condenses there, releasing thereby the latent heat of condensation. The condensate returns back to the evaporator completing the fluid cycle.

A gravity assisted heat pipe of special form, shown in figure(2), is constructed as a unit of the proposed system. 'l'he heat pipe is made from commercial steel tubing of $1-i n c h$ nominal diameter. It is assembled of two parts. The Eirst of them is the evaporator section while the second is the condenser section. The main special features of the heat pipe which make it able to fulfill the above requirements could be summarized as follows :

1- The evaporator is formed as half ring with a mean diameter of $300 \mathrm{~mm}$. This form is chosen so that every pair of heat pips makes a ring and all the rings are vertically located above each other within the absorber core. The outlets of each two successive rings make $90^{\circ}$ to one another.

The circular form of the evaporator enables minimizing the absorber volume. The evaporator-pairs with its vertical arrangement makes it possible (by using only one distributing equipment) to spread the weak solution in a film form on the outer surface of the successive rings. The absorbing liquid falls from one $x i n g$ to the next and creates the required liguid
film for the mass transfer mechanism.

2. The condenser section, shown in figure-2 is made from the and thickness helical fins with an outer diameter of $60.0 \mathrm{~mm}$. of heat trans of $0.7 \mathrm{~mm}$., to increase the overall coefficient condenser has from the outer tube surface to the air. The gravity back to inclination of $10^{\circ}$ to return the condensate by condenser and the location of the The proposed form of the is chosen to increase the natural heat pipes above each other the heat transfer athich inturn improves absorber more compact. 
The ends of both evaporator and condenser are closed by ? welding end cups. The condenser end is provided with a $1 / 8$ " diameter filling copper tube. 'The filling of the heat pipe is carried out on a rig specially developed for this purpose. Figure (3) shows a layout of the heat pipe filling rig.

F-11 is chosen as the working medium. 'The vapor pressure of F-11 lies within the atmospheric pressure for the system operating temperatures. 'That has two advantages. 'Ihe first is that the tightness could be guaranteed for a long time while the second the thickness of the commercial tubes can safely withstand the pressure difference.

\section{HEAT PIPE TESTING RIG}

In order to determine the heat transport capability of the proposed gravity assisted heat pipe, a special test rig as shown in figure-4 is developed. The test procedure aims to investigate the dependence of the transported heat flux upon the operating boundary conditions. The considered parameters are, temperatures of the both hot and the cold mediums, ('T' ${ }_{\mathbf{H}}$ ') and $\left(\mathrm{T}_{\mathbf{C M}}\right.$ ) respectively, the evaporator filling ratio (f), which represents the ratio of the refrigerant-volume to that volume of the refrigerant required for the complete filling of the evaporator, and the hot medium mass flow rate ( ${ }_{\text {HM }}$ ) which influences the falling film characteristics.

As it is above-mentioned, the atmospheric air cools the heat pipe condenser by natural convection, meanwhile hot water is used in the experiments to simulate the hot ammonia-water solution. Simulating the absorbing solution by hot water has no significant error in the experimental results, since the main resistance for the heat transfer mechanism is that of the free convection on the condenser outer surface.

The test rig has the following features and facilities as shown in figure (4):

1. A thermally isolated chamber (10), within which the evaporator section installed. The thermal insulation is used to minimize the heat transmission from the chamber walls. That can guarantee the assumption that the heat flow takes place only through the heat pipe to the air.

2. In order to investigate the performance under different condenser temperatures, a thermally insulated rooll is made to contain the condenser section (13). An air heater (12) with thermostat (14) is installed in this room to maintain the air. temperature (' $\left.r_{\mathbf{3}}\right)$ at the required level.

3. The hot fluid is distributed uniformly along the evaporator section by using a special arrangement (6), which layed over the evaporator within the insulated chamber (10). While under the evaporator a hot fluid collecting dish (7) is used. to gather the hot fluid which falls from the evaporator. 
4. The temperatures of the hot fluid at its inlet of the distributing equipment and at the outlet from the collecting dish (') are measured ('T) and (' $\left.{ }_{2}\right)$ respectively. I'he difference between these two temperatures gives the temperature drop due to the heat loss through the heat pipe.

5. The hot fluid is collected, within a graduated glass cylinder (9), during the time of the experiment in order to evaluate the mean mass flow rate of the hot fluid.

6. In order to insure constant flow rate of the hot fluid, a constant level tank ( 1 ) is used as supply for the hot fluid. The tank level is controlled by using float valve (2).

7. The hot fluid flow rate changed to the desired value using the valve (3). The test rig is supplied with a flow-meter (4) in order to adjust the hot fluid mass flow rate.

8. The temperature of the hot fluid is inaintained constant by using the thermostatic heater (5). The control unit has sensitivity of $0.1{ }^{\circ} \mathrm{C}$. The hot fluid temperature has been changed to study its effect on the operating performance.

4.

\section{RESULTS}

It was aimed to investigate the performance of the heat pipe, which is specially constructed to transmit heat from the absorber core, by an absorption refrigerator, naturally convective to the air. This process should be guaranteed for both falling film and axising bubble absorption concepts. The investigation were carried out under the actual operating conditions. The operating parameters which were considered during this investigation are; heat-pipe capability, evaporator filling ratio, condenser temperature, (which is the ambient air temperature) and the evaporator temperature (which is the not fluid temperature). The experimental investigation was carried out with the aid of the above mentioned test-rig, to clarify the dependence of the heat pipe capability upon the other operating parameters. Many sets of experiments are carried out. During each of the experiment-set:s one of the independent parameters has been changed within the range expected for the actual. operation. The evaporator filling ratio has been varied form $5 \%$ to $30 \%$ with $5 \%$ step wise. The experiments were carried out only within this range since it is recommended by references [1] and [3].

Figure (5) shows the dependence of the heat-pipe capability on the evaporator filling-ratio under two different values of the condenser temperatures. From this figure it could be seen that in the range from 10 to $30 \%$, for the filling ratio the heat pipe capability is nearly constant for air temperature of $30^{\circ} \mathrm{C}$. For filling ratios under $10 \%$ the heat Elux decreases severely with the decrease of the filling ratio. In this range the burn out phenomena appears. Increasing the air temperature, which leads to the decrease of the difference between the condenser and the evaporator temperatures, delays the appearance of this phenomena and perhaps diminishes it totally. 
Figures $(6,7,8)$ show the results of the t.est runs during which the hot fluid and the air temperalures are varied. From these figures it could be seen that he heat pipe capability depends strongly upon the temperature difference between the hot fluid and the air. 'lhis effect is more significant by higher refrigerant evaporator filling ratio than that by lower values for it.

It could be seen from figures (6, 7, 6) lhat, the hot fluid flow rate has a slight effect on the heat pipe capability. It is found that increasing the flow rate of the hot fluid three times increases the heat pipe capability only about $20 \%$ by low temperature difference between hot and cold media and about $24 \%$ by high temperature difference. This is because of the consideration of heat lransport mechanism (neglecting the mass transport, absorption, one).

\section{CONCLUSI ONS}

- Natural convection cooling of the absorber of an autonomous solar refrigerator could be achieved by using the above described heat pipe concept.

- The heat transferred by the heat pipe depends essentially upon the temperature difference between the condenser and the evaporator.

- The evaporator filling degree for the heat pipe must be higher than $20 \%$ to avoid the burn out phenomenon.

\section{REFERENCES}

L1j M. Clerx and G. J. 'l'rezek, "Perlormance of an AquaAmmonia Absorption solar Refrigerator at Sub-Freezing Evaporator Conditions," Solar Energy, vol. 39, 1987.

[2] P. Dunn and D. A. Reay," Heat pipes," Pergamon press 1982

(3) Mehmet Akyurt," Development of Heat pipes for solar water Heaters," Solar Energy, Vol 32, No. 5, 1984.

[4] G. Rice and J. D. Jennings, " Heat Pipe billing," proceedings of the 1st. International Heat pipe Conference, Stuttgert, 1973 .

[5] K. Neighs and T. Saw ada;" "Heat Transfer performance of Inclined 'l'wo-phase T'hermosyphon," Int. J. Heat and Mass Transfer, Vol. 26 no. 8, 1983.

[6] J. V. MC Allan and C. Bassani, "condenser instability in pressure-controlled heat pipes ", Wärme und stoffübertragung, vol. 19, 1985. 


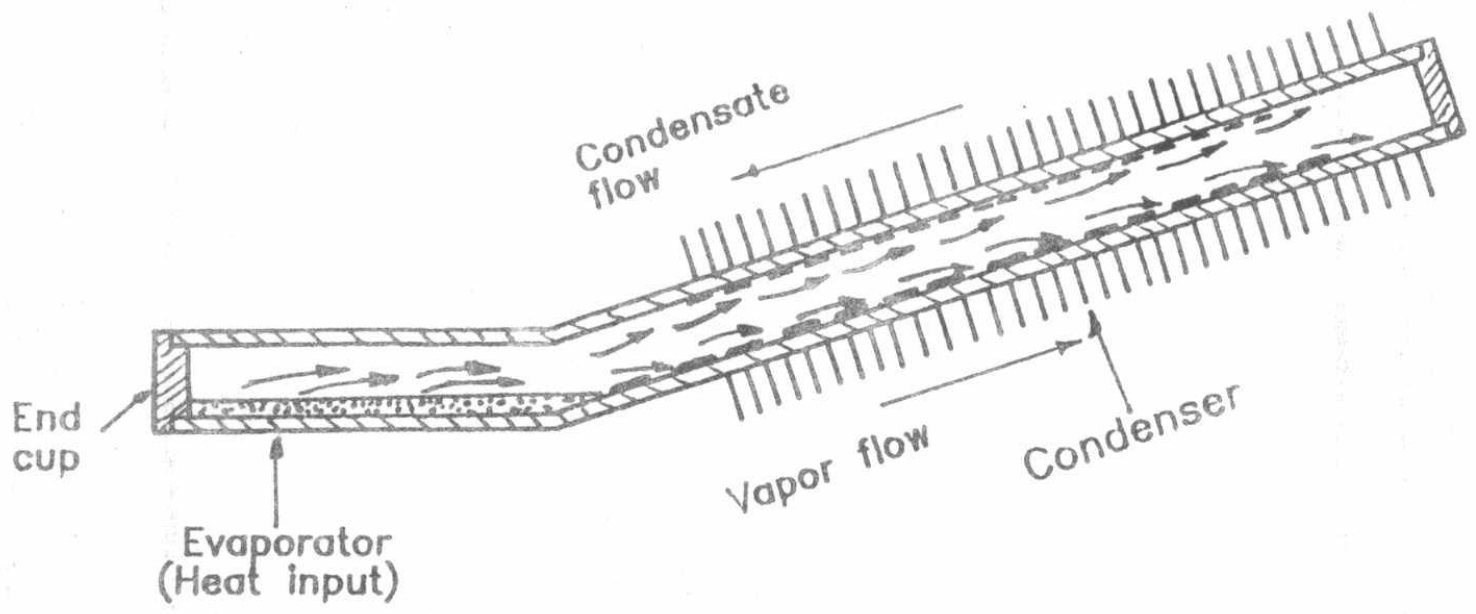

Fig. (1) Schematic Diagram of the Gravity-Assisted Heat-Pipe

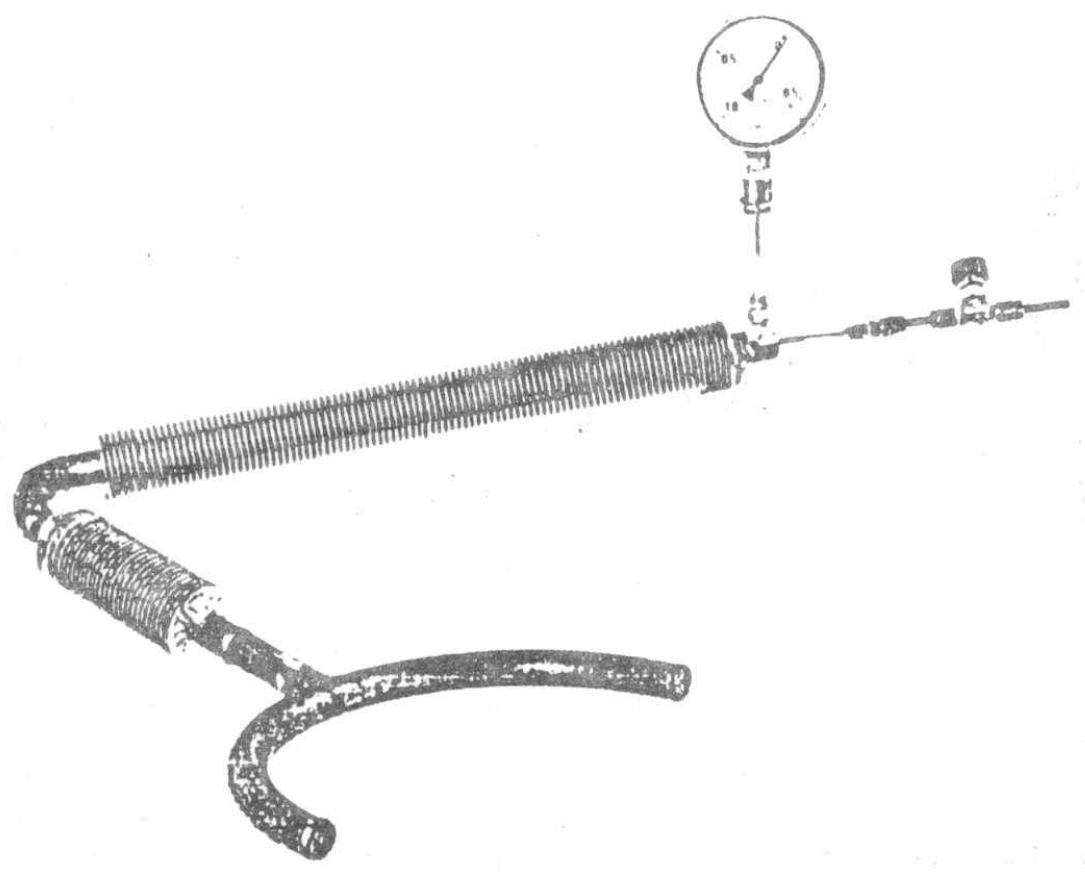

F'ig. (2) The Gravity-Assisted Heat-Pipe 


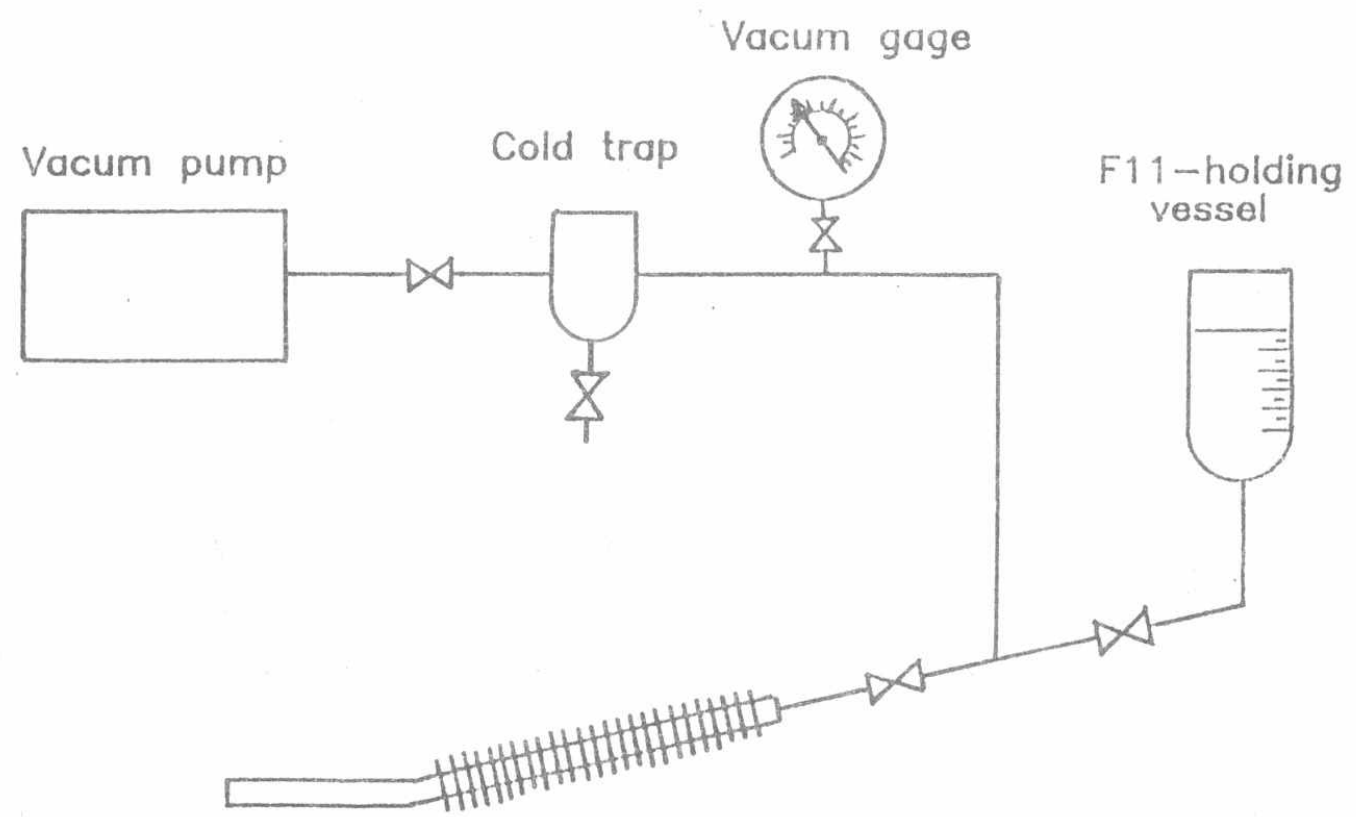

Heat pipe

Fig. (3) Layout of the Heat-pipe Filling Rig 
$\Gamma$
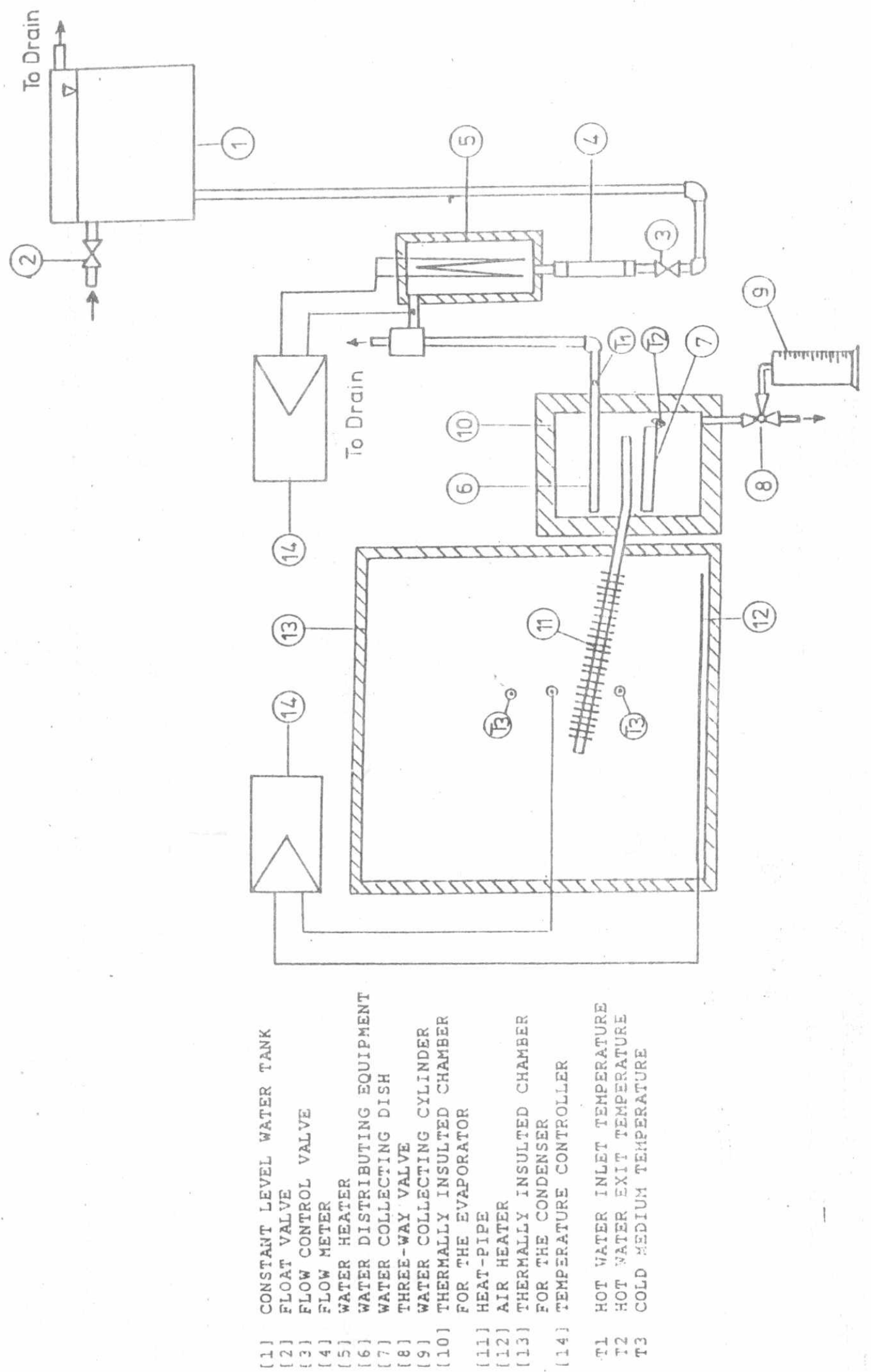

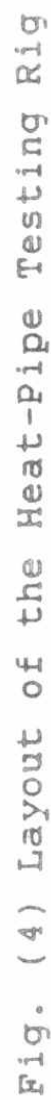




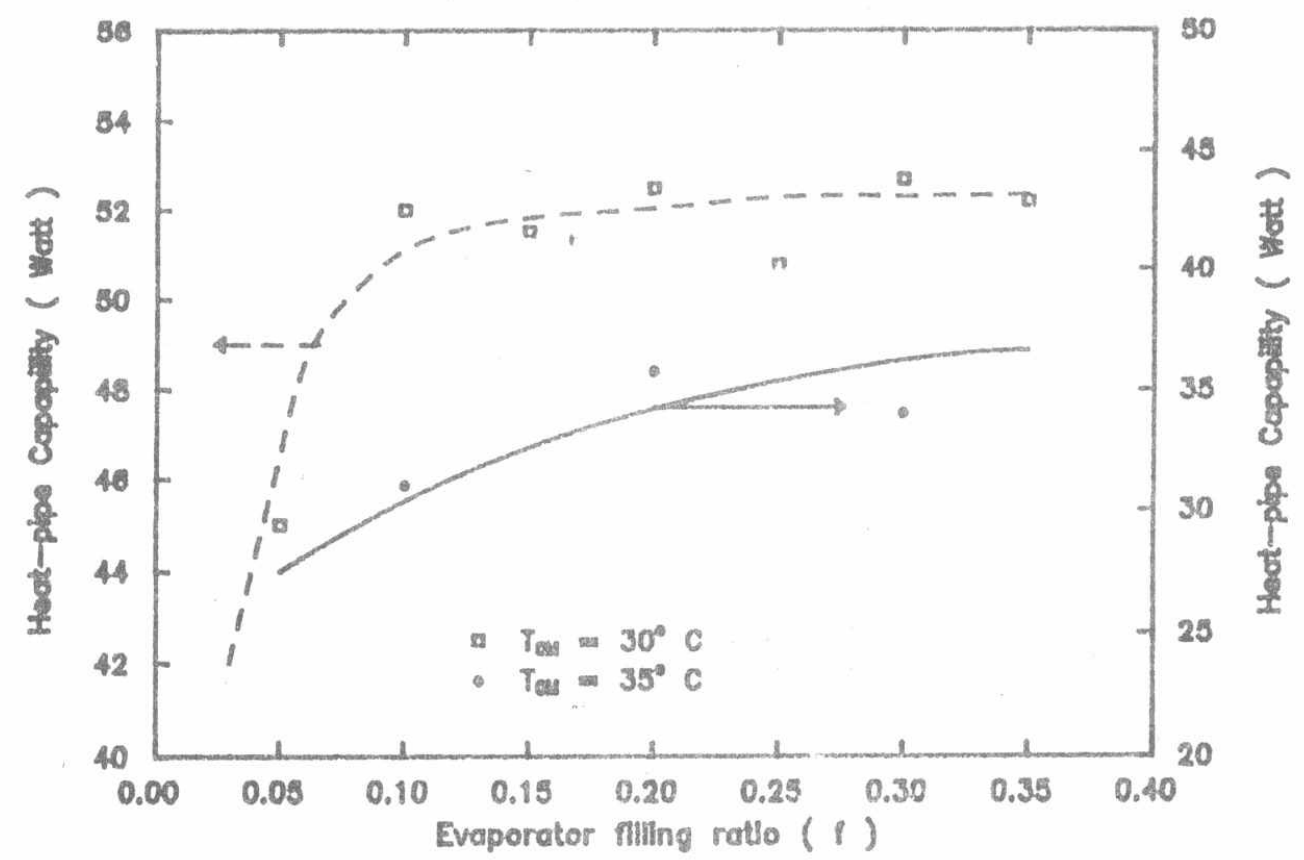

Fig. (5) Dependence of the Heat-Plpe Capapillty Upon

The cooling miedlum temperaur $\left(T_{\mathrm{cas}}\right)$ and the evaporator filling ratio (t) for a hot miedium tempsraur $50^{\circ} \mathrm{C}$

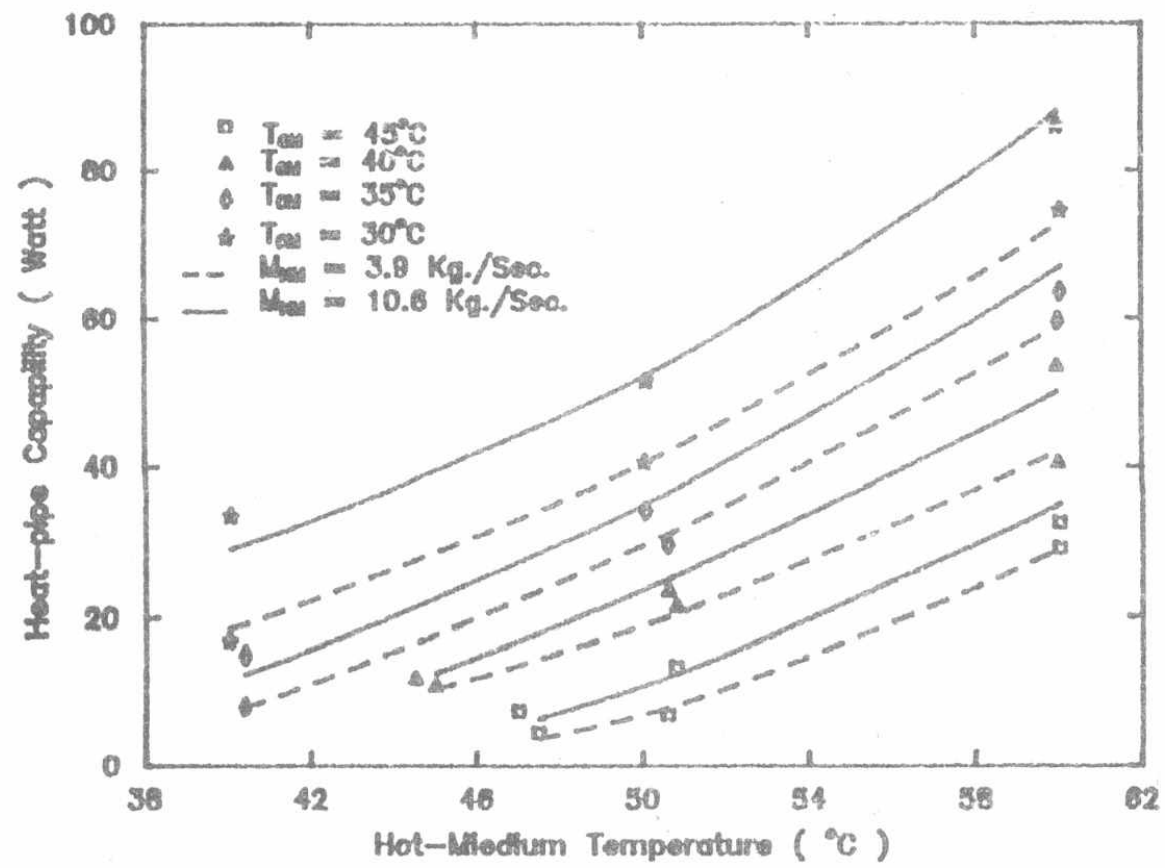

Fig. (6) The Effect of both Hot and Cold Mledium Tomperatures on Heat-Pipe Capopilty for an Evaporator-Fillthig ratio of $10 \%$ 


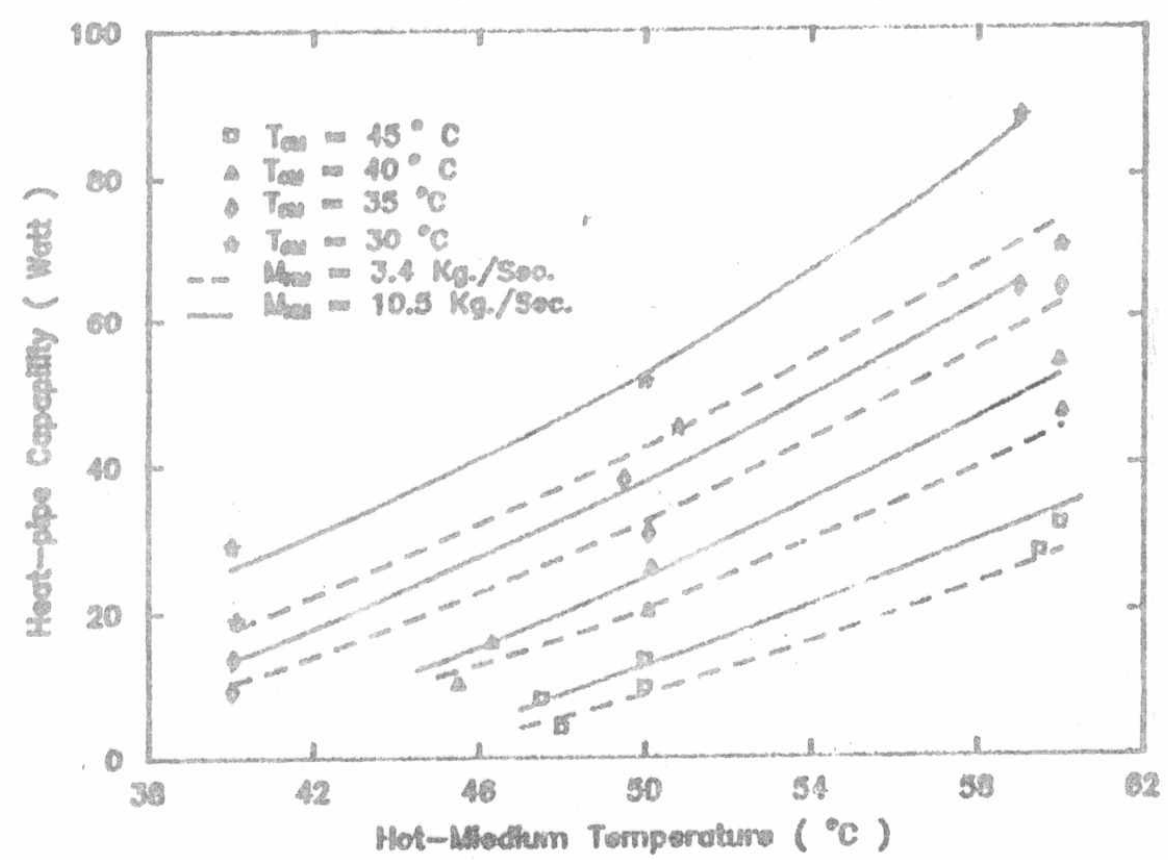

Fig. (7) The Effect of both Hot ond Cold Miedium Temperatuses on Heat-Pipe Copopility for an Eveporator-Fiting ratlo of 20 s

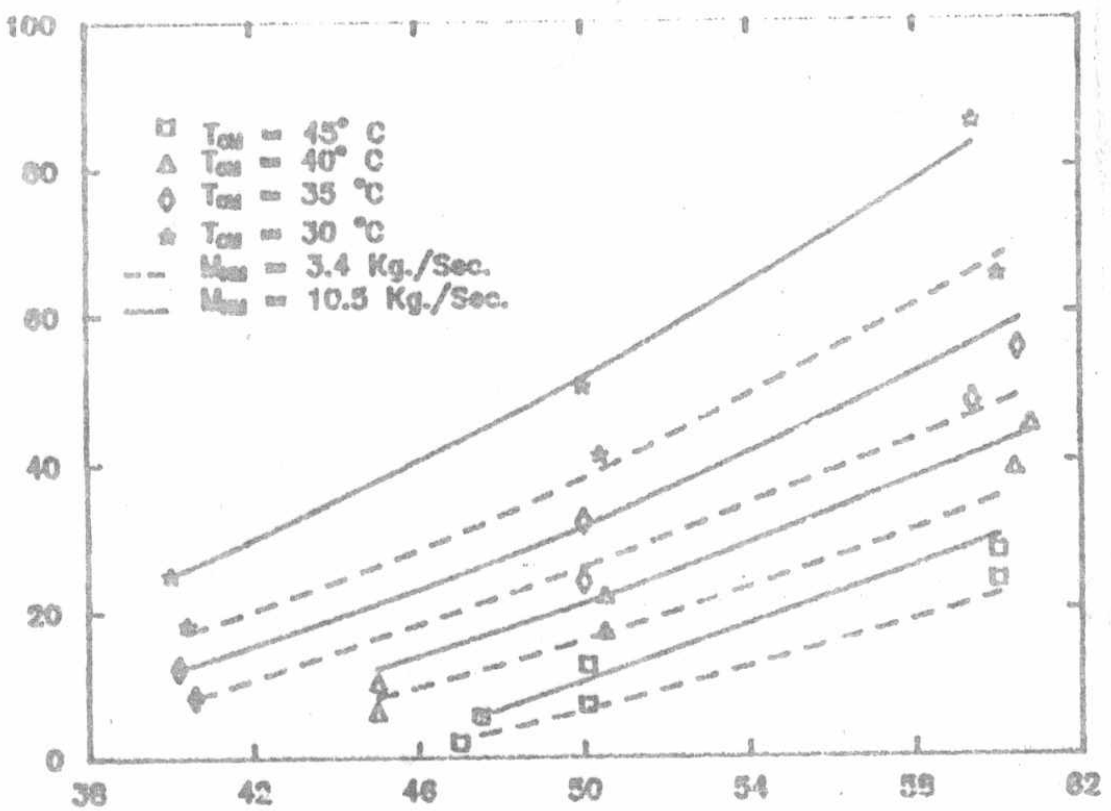

Fig. (8) The Effect of both Hot and Cold Miedium Temperatures on Meat-Pipe Copopiatly for an Evaporator-Filling rotio of 30 \& 